\title{
Random variate generation and connected computational issues for the Poisson-Tweedie distribution
}

This is the peer reviewed version of the following article:

Original:

Baccini, A., Barabesi, L., Stracqualursi, L. (2016). Random variate generation and connected computational issues for the Poisson-Tweedie distribution. COMPUTATIONAL STATISTICS, 31(2), 729-748 [10.1007/s00180-015-0623-5].

Availability:

This version is availablehttp://hdl.handle.net/11365/983552

since 2018-09-20T17:03:37Z

\section{Published:}

DOI:10.1007/s00180-015-0623-5

Terms of use:

Open Access

The terms and conditions for the reuse of this version of the manuscript are specified in the publishing policy. Works made available under a Creative Commons license can be used according to the terms and conditions of said license.

For all terms of use and more information see the publisher's website.

(Article begins on next page) 


\title{
Random variate generation and connected computational issues for the Poisson-Tweedie distribution
}

\author{
A. Baccini $^{1}$, L. Barabesi $^{1}$, L. Stracqualursi ${ }^{2}$ \\ ${ }^{1}$ Department of Economics and Statistics, University of Siena, \\ P.zza S.Francesco 7, 53100 Siena, Italy \\ ${ }^{2}$ Department of Statistical Sciences "Paolo Fortunati”, University of Bologna, \\ Via Belle Arti 41, 40126 Bologna, Italy
}

\begin{abstract}
After providing a systematic outline of the stochastic genesis of the Poisson-Tweedie distribution, some computational issues are considered. More specifically, we introduce a closed form for the probability function, as well as its corresponding integral representation which may be useful for large argument values. Several algorithms for generating PoissonTweedie random variates are also suggested. Finally, count data connected to the citation profiles of two statistical journals are modeled and analyzed by means of the PoissonTweedie distribution.
\end{abstract}

Key words. Mixture Poisson distribution; Compound Poisson distribution; Random variate generation; Journal citation profile data.

1. Introduction. The Poisson-Tweedie integer-valued distribution has been introduced independently by Gerber (1991) - as the generalized Negative Binomial distribution - and Hougaard et al. (1997) - as the P-G distribution (where the acronym emphasizes its stochastic representation as a mixture Poisson, see Section 2 for more details). At first, the distribution seems to be named 'Poisson-Tweedie' by Kokonendji et al. (2004) - even if Johnson et al. (2005, p.480) refer to it as 'Tweedie-Poisson' in their encyclopedia. The denomination is obviously due to the strong connection of this integer-valued law with the absolutelycontinuous Tweedie distribution introduced by Hougaard (1986) on the basis of the seminal proposal by Tweedie (1984) - see Section 2 of the present paper. The Poisson-Tweedie is a very flexible model and contains as special cases classical families such as the Poisson and the Negative Binomial, as well as large families such as the Generalized Poisson Inverse Gaussian and the Poisson-Pascal - and even the Discrete Stable family (see e.g. El-Shaarawi et al., 2011).

By following the notation adopted by El-Shaarawi et al. (2011), the probability generating function (p.g.f.) of the Poisson-Tweedie random variable (r.v.) $X_{\mathrm{PT}}$ is given by

$$
G_{X_{\mathrm{PT}}}(s)=e^{\frac{b}{a}\left[(1-c)^{a}-(1-c s)^{a}\right]},
$$

where $(a, b, c) \in\{]-\infty, 0] \times] 0, \infty[\times[0,1[\} \cup\{] 0,1] \times] 0, \infty[\times[0,1]\}$. It should be remarked that the case $a=0$ may be managed for analytical continuation. In the following, the Poisson-Tweedie r.v. with p.g.f. given by (1) is eventually denoted as $\mathcal{P} \mathcal{T}(a, b, c)$ for convenience. The probability function (p.f.) of the r.v. $X_{\mathrm{PT}}$ is usually computed by means of the recursive algorithm given by El-Shaarawi et al. (2011). Many properties of the 
distribution may be found in the papers by Hougaard et al. (1997), Kokonendji et al. (2004) and El-Shaarawi et al. (2011).

As to the practical applications, the Poisson-Tweedie distribution is very useful for modeling overdispersed count data arising in very different settings. As an example, Hougaard et al. (1997) provide an analysis of clinical data involving epileptic seizure frequency, while El-Shaarawi et al. (2011) consider environmental studies based on bacteria counts. In turn, the distribution is adopted by Kokonendji et al. (2004) in connection of car insurance claim data and by Hao-Chun Chuang and Oliva (2014) for analyzing retail demand data. As a final example, Esnaola et al. (2013) recently consider the Poisson-Tweedie distribution for modeling count data in RNA sequencing.

The present paper is focused on some theoretical and computational issues connected to the Poisson-Tweedie distribution. More precisely, a closed and simple expression - suitable for the evaluation of the p.f. - is initially introduced. This expression may be helpful in order to save time and achieve a better accuracy in the computation of maximum likelihood estimates, especially when the sample size is large. Alternatively, an integral expression for the p.f. is proposed. This expression may be convenient when the p.f. has to be computed for large argument values. Subsequently, some algorithms for random variate generation are suggested on the basis of a discussion of the stochastic geneses of the distribution. The comparison of the algorithms is theoretically and practically performed and some suggestions on the most efficient generators are provided.

The paper is organized as follows. In Section 2, the representation of the Poisson-Tweedie distribution as either a mixture Poisson distribution or a compound Poisson distribution is considered. In Section 3, the expressions of the p.f. - suitable for computation purposes - are given. Section 4 contains the algorithms for random variate generation, as well as their comparison in terms of some performance benchmarks. Finally, a scientometric application is considered in Section 5 by analyzing the citation profiles of two leading Italian statistical journals.

2. Stochastic genesis of the Poisson-Tweedie distribution. In order to implement the random variate generators which will be introduced in Section 4, it is useful to consider the nature of the Poisson-Tweedie distribution as a mixture Poisson or a compound Poisson. The following results - mostly known, even if scattered in literature - are given here in a homogeneous notation, which should clarify the various distributional equivalences.

The Poisson-Tweedie distribution may be stochastically represented as a mixture Poisson distribution, with a mixturing absolutely-continuous Tweedie r.v. Indeed, Hougaard (1986) and Hougaard et al. (1997) give the Laplace transform of the absolutely-continuous Tweedie r.v. $X_{\mathrm{T}}$ with following parameterization

$$
L_{X_{\mathrm{T}}}(t)=e^{\frac{\delta}{\gamma}\left[\theta^{\gamma}-(\theta+t)^{\gamma}\right]}, \operatorname{Re}(t)>0,
$$

where $(\gamma, \delta, \theta) \in\{]-\infty, 0[\times] 0, \infty[\times] 0, \infty[\} \cup\{] 0,1] \times] 0, \infty[\times] 0, \infty[\}$. We eventually denote this r.v. by $\mathcal{T}(\gamma, \delta, \theta)$. By reparametrizing in such a way that $\gamma=a, \delta=b c^{a}$ and $\theta=(1-c) / c$ and since on the basis of (1) and (2) the p.g.f. of the Poisson-Tweedie may be rewritten as

$$
G_{X_{\mathrm{PT}}}(s)=e^{\frac{b c^{a}}{a}\left[\left(\frac{1-c}{c}\right)^{a}-\left(\frac{1-c}{c}+1-s\right)^{a}\right]}=L_{X_{\mathrm{T}}}(1-s),
$$

it promptly follows the stochastic representation (see also Hougaard et al., 1997) 


$$
\mathcal{P} \mathcal{T}(a, b, c) \stackrel{\mathcal{L}}{=} \mathcal{P}\left(\mathcal{T}\left(a, b c^{a},(1-c) / c\right)\right),
$$

where $\mathcal{P}(\mu)$ denotes a Poisson r.v. with parameter $\mu$.

As to the absolutely-continuous Tweedie distribution, for $\gamma \in] 0,1]$ the r.v. $X_{\mathrm{T}}$ can be rephrased as an exponentially-tilted Positive Stable r.v. Indeed, let us consider the absolutelycontinuous Positive Stable r.v. $X_{\mathrm{PS}}$ with Laplace transform given by

$$
L_{X_{\mathrm{PS}}}(t)=e^{-\lambda t^{\gamma}}, \operatorname{Re}(t)>0,
$$

where $(\gamma, \lambda) \in\{[0,1] \times] 0, \infty[\}$. In the following, this Positive Stable r.v. is also denoted by $\mathcal{P} \mathcal{S}(\gamma, \lambda)$. By reparametrizing in such a way that $\lambda=\delta / \gamma$, it follows that

$$
L_{X_{\mathrm{T}}}(t)=\frac{L_{X_{\mathrm{PS}}}(\theta+t)}{L_{X_{\mathrm{PS}}}(\theta)}
$$

and hence the absolutely-continuous Tweedie distribution may be actually seen as an exponentially-tilted Positive Stable distribution (see also Hougaard, 1986). In contrast, for $\gamma \in]-\infty, 0[$, the Laplace transform of the absolutely-continuous Tweedie distribution may be rewritten as

$$
L_{X_{\mathrm{T}}}(t)=e^{-\frac{\delta \theta^{\gamma}}{\gamma}\left[\left(\frac{\theta+t}{\theta}\right)^{\gamma}-1\right]},
$$

i.e. the r.v. $X_{\mathrm{T}}$ may be expressed as the compound of a Poisson distribution, with parameter $\left(-\delta \theta^{\gamma} / \gamma\right)$, of i.i.d. Gamma r.v.'s with shape parameter $(-\gamma)$ and scale parameter $(1 / \theta)$ (see also Aalen, 1992). More precisely, if $\mathcal{G}(k, \sigma)$ denotes a Gamma r.v. with shape parameter $k$ and scale parameter $\sigma$, owing to the reproductive property of the Gamma distribution, the absolutely-continuous Tweedie r.v. may be stochastically represented as

$$
\mathcal{T}(\gamma, \delta, \theta) \stackrel{\mathcal{L}}{=} \mathcal{G}\left(-\gamma \mathcal{P}\left(-\delta \theta^{\gamma} / \gamma\right), 1 / \theta\right)
$$

(with the assumption that the Gamma r.v. with a null shape parameter degenerates to the Dirac mass at zero). Hence, for $a \in]-\infty, 0[$, it also follows from (3)

$$
\mathcal{P} \mathcal{T}(a, b, c) \stackrel{\mathcal{L}}{=} \mathcal{P}\left(\mathcal{G}\left(-a \mathcal{P}\left(-b(1-c)^{a} / a\right), c /(1-c)\right)\right),
$$

where the two Poisson r.v.'s involved in (4) are independent.

Finally, when $a=0$ the Poisson-Tweedie r.v. reduces to a Negative Binomial r.v., say $X_{\mathrm{NB}}$, with p.g.f.

$$
G_{X_{\mathrm{NB}}}(s)=\left(\frac{1-c}{1-c s}\right)^{b},
$$

which is also denoted by $\mathcal{N B}(b, c)$ in the following. In addition, for $\gamma=0$ the absolutelycontinuous Tweedie distribution reduces to a Gamma distribution with parameters $\delta$ and $\theta$. In such a case, the representation follows

$$
\mathcal{P} \mathcal{T}(0, b, c) \stackrel{\mathcal{L}}{=} \mathcal{N B}(b, c) \stackrel{\mathcal{L}}{=} \mathcal{P}(\mathcal{G}(b, c /(1-c))) .
$$

A further stochastic representation of the Poisson-Tweedie distribution may be obtained in terms of a compound Poisson distribution. Actually, for $a \in] 0,1]$, the Poisson-Tweedie r.v. is a compound Poisson r.v. with a compounding of a geometric down-weighting Sibuya r.v. Indeed, let $X_{\mathrm{SI}}$ be a Sibuya r.v. (as named by Devroye, 1993) with p.g.f. 


$$
G_{X_{\mathrm{SI}}}(s)=1-(1-s)^{\gamma},
$$

where $\gamma \in] 0,1]$. The Sibuya distribution is a special case of the (shifted) Negative Binomial Beta distribution introduced by Sibuya (1979) with parameters given by $1, \gamma$ and $(1-\gamma)$. In the following, the Sibuya r.v. is also denoted by $\mathcal{S I}(\gamma)$. On the basis of the findings by Sibuya (1979), if $\mathcal{B}(\phi, \varphi)$ represents a Beta r.v. with shape parameters $\phi$ and $\varphi$, the Sibuya r.v. has the following stochastic representation

$$
\mathcal{S} \mathcal{I}(\gamma) \stackrel{\mathcal{L}}{=} 1+\mathcal{N} \mathcal{B}(1, \mathcal{B}(1-\gamma, \gamma)) \stackrel{\mathcal{L}}{=} 1+\mathcal{P}(\mathcal{G}(1,1) \mathcal{G}(1-\gamma, 1) / \mathcal{G}(\gamma, 1)),
$$

where the Exponential and the two Gamma r.v.'s involved in the previous expression are assumed to be independent. In this case, the geometric down-weighting Sibuya r.v. $X_{\mathrm{DSI}}$ displays the p.g.f.

$$
G_{X_{\mathrm{DSI}}}(s)=1-G_{X_{\mathrm{SI}}}(\beta)+G_{X_{\mathrm{SI}}}(\beta s)=1+(1-\beta)^{\gamma}-(1-\beta s)^{\gamma},
$$

where $(\gamma, \beta) \in\{[0,1] \times] 0,1]\}$ (for more details, see Zhu and Joe, 2009). The geometric down-weighting Sibuya r.v. is also denoted by $\mathcal{D S I}(\gamma, \beta)$. Therefore, if $\mathcal{U}$ represents a Uniform r.v. on $[0,1]$, the following representation holds

$$
\mathcal{D S I}(\gamma, \beta) \stackrel{\mathcal{L}}{=} I_{\mathbb{R}^{+}}\left(\beta^{\mathcal{S I}(\gamma)}-\mathcal{U}\right) \mathcal{S I}(\gamma),
$$

where the r.v. $\mathcal{U}$ is independent of the r.v. $\mathcal{S} \mathcal{I}(\gamma)$, while $I_{B}$ is the usual indicator function of a set $B$. Finally, by reparametrizing in such a way that $\gamma=a$ and $\beta=c$, from (1) it turns out that

$$
G_{X_{\mathrm{PT}}}(s)=e^{\frac{b}{a}\left[\left(1+(1-c)^{a}-(1-c s)^{a}\right)-1\right]},
$$

i.e. a Poisson compounding of a geometric down-weighting Sibuya r.v. is actually achieved. Hence, the stochastic representation holds

$$
\mathcal{P} \mathcal{T}(a, b, c) \stackrel{\mathcal{L}}{=} \sum_{i=1}^{\mathcal{P}(b / a)} \mathcal{D} \mathcal{S I}_{i}(a, c),
$$

where the $\mathcal{D S I}_{i}(a)$ 's are i.i.d. geometric down-weighting Sibuya r.v.'s, which are in turn independent of $\mathcal{P}(b / a)$.

When $a \in]-\infty, 0[$, from (1) it promptly follows that

$$
G_{X_{\mathrm{PT}}}(s)=e^{-\frac{b(1-c)^{a}}{a}\left[\left(\frac{1-c s}{1-c}\right)^{a}-1\right]},
$$

i.e. a Poisson compounding of a Negative Binomial r.v. with parameters $(-a)$ and $c$ is obtained. Hence, the following representation holds

$$
\mathcal{P} \mathcal{T}(a, b, c) \stackrel{\mathcal{L}}{=} \sum_{i=1}^{\mathcal{P}\left(-b(1-c)^{a} / a\right)} \mathcal{N B}_{i}(-a, c),
$$

where the $\mathcal{N B}_{i}(-a, c)$ 's are i.i.d. Negative Binomial r.v.'s, which are in turn independent of $\mathcal{P}\left(-b(1-c)^{a} / a\right)$. Owing to the reproductive property of the Negative Binomial, the previous expression reduces to

$$
\mathcal{P} \mathcal{T}(a, b, c) \stackrel{\mathcal{L}}{=} \mathcal{N B}\left(-a \mathcal{P}\left(-b(1-c)^{a} / a\right), c\right),
$$


which is stochastically equivalent to (4) by considering (5). Finally, when $a=0$ the representation (5) is in turn achieved.

3. Easy-computable expressions for the p.f. First, it is worth noting that the p.f. corresponding to the p.g.f. (1) may be obtained as a finite sum. Indeed, from Result 1 in the Appendix, it turns out to be

$$
p_{X_{\mathrm{PT}}}(k)=e^{\frac{b}{a}\left[(1-c)^{a}-1\right]}(-c)^{k} \sum_{m=0}^{k} \frac{(b / a)^{m}}{m !} \sum_{j=0}^{m}(-1)^{j}\left(\begin{array}{c}
m \\
j
\end{array}\right)\left(\begin{array}{c}
a j \\
k
\end{array}\right) I_{\mathbb{N}}(k) .
$$

In addition, from Result 2 in the Appendix, for $a \in] 0,1]$ it also follows that

$$
p_{X_{\mathrm{PT}}}(k) \leq \frac{b}{a}\left(1+\frac{b}{a}\right) e^{\frac{b}{a}\left[(1-c)^{a}+1\right]} c^{k} k^{-a-1} .
$$

Moreover, by adopting the expression given in Result 2 for $p_{X_{\mathrm{PT}}}$, for a fixed $k_{*}$ the following simple approximation of $p_{X_{\mathrm{PT}}}$ holds

$$
p_{X_{\mathrm{PT}}}^{*}(k)=e^{\frac{b}{a}(1-c)^{a}}(-c)^{k} \sum_{m=0}^{k^{*}}(-1)^{m}\left(\begin{array}{c}
a m \\
k
\end{array}\right) \frac{(b / a)^{m}}{m !} .
$$

We have numerically assessed that $k_{*}=2$ usually suffices for obtaining an adequate approximation for a large $k$ - which may avoid the computational burden involved in the evaluation of (9) in such a case.

Incidentally, it is interesting to remark that for $a \in] 0,1]$ the Poisson-Tweedie r.v. may be rephrased as an exponentially-tilted Discrete Stable r.v. - i.e. the integer-valued counterpart of an exponentially-tilted Stable r.v. Indeed, let us notice that the p.g.f. of the Discrete Stable r.v. $X_{\mathrm{DS}}$ of parameters $\gamma$ and $\lambda$ is given by

$$
G_{X_{\mathrm{DS}}}(s)=e^{-\lambda(1-s)^{\gamma}},
$$

where $(\gamma, \lambda) \in\{] 0,1] \times] 0, \infty[\}$ (for more details on this heavy-tailed distribution, see e.g. Marcheselli et al., 2008). In the following, this r.v. is also denoted as $\mathcal{D S}(\gamma, \lambda)$. Hence, by reparametrizing in such a way that $\gamma=a$ and $\lambda=b / a$, on the basis of expression (1) it follows that

$$
G_{X_{\mathrm{PT}}}(s)=\frac{G_{X_{\mathrm{DS}}}(c s)}{G_{X_{\mathrm{DS}}}(c)} .
$$

Thus, if $p_{X_{\mathrm{DS}}}$ represents the p.f. of the Discrete Stable r.v. it turns out that

$$
p_{X_{\mathrm{PT}}}(k)=e^{\frac{b}{a}(1-c)^{a}} c^{k} p_{X_{\mathrm{DS}}}(k),
$$

i.e. an exponentially-tilted Discrete Stable r.v. with tilting parameter $c$ is actually achieved. From expressions (9) and (11), a closed form for the p.f. of the Discrete Stable r.v. $\mathcal{D S}(\gamma, \lambda)$ can be obtained as a by-product, i.e.

$$
p_{X_{\mathrm{DS}}}(k)=e^{-\frac{b}{a}}(-1)^{k} \sum_{m=0}^{k} \frac{(b / a)^{m}}{m !} \sum_{j=0}^{m}(-1)^{j}\left(\begin{array}{c}
m \\
j
\end{array}\right)\left(\begin{array}{c}
a j \\
k
\end{array}\right) I_{\mathbb{N}}(k) .
$$


For further discussion of integer-valued exponentially-tilted distributions related to the Discrete Stable distribution see Barabesi and Pratelli (2014a).

As previously remarked, expression (9) is not obviously convenient for large values of $k$, even if its computation solely requires a finite summation. The recursive expression for $p_{X_{\mathrm{PT}}}$ provided by El-Shaarawi et al. (2011) actually involves the same drawback. In such a case, the p.f. $p_{X_{\mathrm{PT}}}$ may be alternatively computed by adopting a generalization of the Inversion Theorem. Indeed, by following Barabesi and Pratelli (2014b), if $X$ is an integer-valued r.v. with p.f. $p_{X}$ and $g$ is a measurable function defined on $\mathbb{Z}$ such that $\mathrm{E}[|g(X)|]<\infty$, then it holds

$$
p_{X}(k)=\frac{1}{2 \pi g(k)} \int_{-\pi}^{\pi} e^{-\mathbf{i} t k} \mathrm{E}\left[g(X) e^{\mathbf{i} t X}\right] \mathrm{d} t
$$

for $k \in \mathbb{Z}$ and $g(k) \neq 0$ and where $\mathbf{i}$ represents the imaginary unit. In the case of the PoissonTweedie distribution, by selecting $g(k)=q^{k}$ for a given $\left.q \in\right] 0,1 / c[$, the previous expression gives rise to

$$
\begin{aligned}
p_{X_{\mathrm{PT}}}(k) & =\frac{e^{\frac{b}{a}(1-c)^{a}}}{2 \pi} q^{-k} \int_{-\pi}^{\pi} e^{-\mathbf{i} t k-\frac{b}{a}\left(1-c q e^{\mathrm{i} t}\right)^{a}} \mathrm{~d} t \\
& =\frac{e^{\frac{b}{a}(1-c)^{a}}}{\pi} q^{-k} \int_{0}^{\pi} e^{-b \rho_{a, c q}(t) \cos \left[\psi_{a, c q}(t)\right]} \cos \left[t k-b \rho_{a, c q}(t) \sin \left(\psi_{a, c q}(t)\right)\right] \mathrm{d} t,
\end{aligned}
$$

where

$$
\rho_{a, d}(t)=\frac{1}{a}\left(1+d^{2}-2 d \cos t\right)^{a / 2},
$$

and

$$
\psi_{a, d}(t)=a \arctan \frac{d \sin t}{1-d \cos t}
$$

It is at once apparent that expression (12) reduces to the usual Inversion Theorem for $q=1$, while a different and suitable choice of $q$ may lead to a faster and more accurate evaluation of $p_{X_{\mathrm{PT}}}$. Indeed, for given $a$ and $b$, the parameter $q$ may be chosen in such a way that $c q$ is fixed at a convenient value. In addition, the remarks provided by Dunn and Smyth (2008) for the numerical integration with oscillating integrands may be helpful in this setting. Finally, it should be remarked that (12) implies that the r.v. $\mathcal{P} \mathcal{T}(a, b, c q)$ may be achieved by the exponentially-tilting of the r.v. $\mathcal{P} \mathcal{T}(a, b, c)$ with tilting parameter $q$ - when $q \in] 0,1[$.

4. Random variate generation. As to the computer generation from the Poisson-Tweedie distribution, in principle the mixture Poisson representation (3) should be the cornerstone. Actually, this equivalence in law leads to the following simple algorithm:

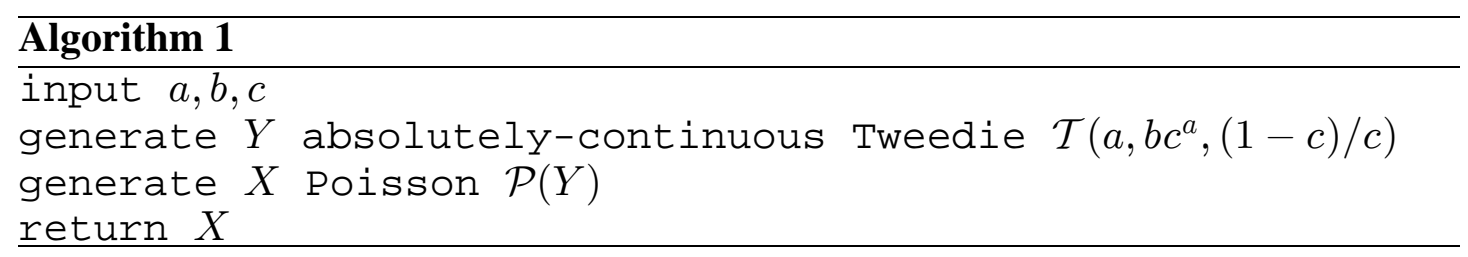


Algorithm 1 is easy-to-implement when $a \in]-\infty, 0$ ] owing to the further representation (4), since Poisson and Gamma variates are commonly available. However, the algorithm may be not convenient when $a \in] 0,1$ ], since in this case the absolutely-continuous Tweedie r.v. is cumbersome to generate and simple and efficient algorithms are not at disposal (see Devroye, 2009, and Hofert, 2011). Hence, the main focus of the present section is devoted to PoissonTweedie variate generation in this parameter range.

When $a \in] 0,1]$, a second procedure may be achieved by means of the compound Poisson representation (8) - and by suitably considering expressions (6) and (7) - which actually leads to the following algorithm:

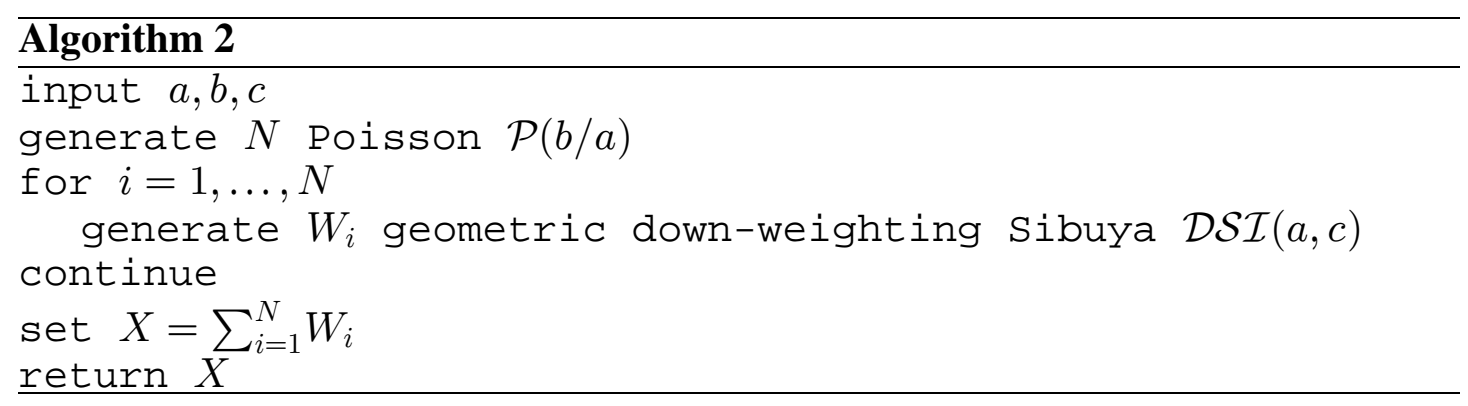

Unfortunately, the geometric down-weighting Sibuya distribution does not possess a reproductive property and hence the cycles in Algorithm 2 cannot be avoided. In addition, it should be remarked that the average number of cycles is given by $(b / a)$ and hence Algorithm 2 is not suitable as $a \downarrow 0$ or $b \rightarrow \infty$. Moreover, since a geometric down-weighting Sibuya variate is obtained on the basis of representations (6) and (7), each cycle actually requires a Geometric variate, a Beta variate and a Uniform variate - alternatively and less conveniently, in turn on the basis of representation (6), each cycle involves a Poisson variate, an Exponential variate, two Gamma variates and a Uniform variate.

As a further option, since in Section 3 it is emphasized that a Poisson-Tweedie r.v. may be seen as an exponentially-tilted Discrete Stable r.v. when $a \in] 0,1]$, a naive algorithm is initially introduced. Let us remind that for the Discrete Stable r.v., Devroye (1993) proved that

$$
\mathcal{D S}(\gamma, \lambda) \stackrel{\mathcal{L}}{=} \mathcal{P}(\mathcal{P} \mathcal{S}(\gamma, \lambda))
$$

Moreover, from the classical Kanter's (1975) representation it turns out that

$$
\mathcal{P S}(\gamma, \lambda) \stackrel{\mathcal{L}}{=}\left(\frac{\sin ((1-\gamma) \pi \mathcal{U})}{\mathcal{G}(1,1) \sin (\gamma \pi \mathcal{U})}\right)^{(1-\gamma) / \gamma}\left(\frac{\lambda \sin (\gamma \pi \mathcal{U})}{\sin (\pi \mathcal{U})}\right)^{1 / \gamma},
$$

where the r.v.'s $\mathcal{G}(1,1)$ and $\mathcal{U}$ are independently distributed. Hence, since from (11) it promptly follows that

$$
p_{X_{\mathrm{PT}}}(k) \leq e^{\frac{b}{a}(1-c)^{a}} c^{k},
$$

and by considering (13) and (14), an acceptance-rejection algorithm for the generation of an exponentially-tilted Discrete Stable variate is given by: 


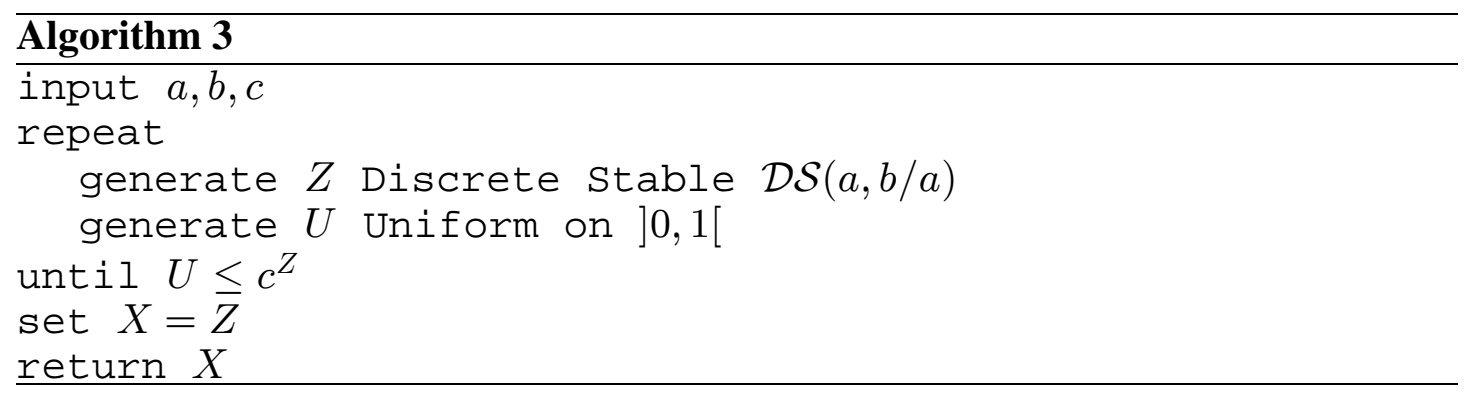

Unfortunately, Algorithm 3 may display a poor performance since the corresponding rejection constant, say $A_{\mathrm{N}}$, is given by

$$
A_{\mathrm{N}}=e^{\frac{b}{a}(1-c)^{a}} .
$$

As usual for an acceptance-rejection algorithm, the rejection constant represents the expected number of iterations to obtain a random variate. Obviously, the best performance is achieved for $c=1$, while the worst performance is obtained when $a \downarrow 0$ or $b \rightarrow \infty$. In any case, the algorithm is not practically acceptable since $A_{\mathrm{N}}=O(\exp (b / a))$. In addition, on the basis of representation (13), the algorithm requires an average of $A_{\mathrm{N}}$ Poisson variates, $2 A_{\mathrm{N}}$ Uniform variates and $A_{\mathrm{N}}$ Exponential variates.

An improved version of the Algorithm 3 may be achieved. Indeed, it is worth remarking that the sum of $m$ i.i.d. Poisson-Tweedie r.v.'s $\mathcal{P} \mathcal{T}(a, b / m, c)$ is a Poisson-Tweedie r.v. $\mathcal{P} \mathcal{T}(a, b, c)$ - i.e. the distribution is actually infinitely divisible with respect to the parameter $b$. Hence, the random generation of $m$ such r.v.'s by means of Algorithm 3 implies a rejection constant given by

$$
A_{\mathrm{IN}}(m)=m e^{\frac{b}{m a}(1-c)^{a}},
$$

which is minimized in $\mathbb{N}$ when $m=m^{*}=\max \left(1, \llbracket(b / a)(1-c)^{a} \rrbracket\right)$ and where $\llbracket \cdot \rrbracket$ represents the rounding function. Hence, the following improved algorithm may be considered:

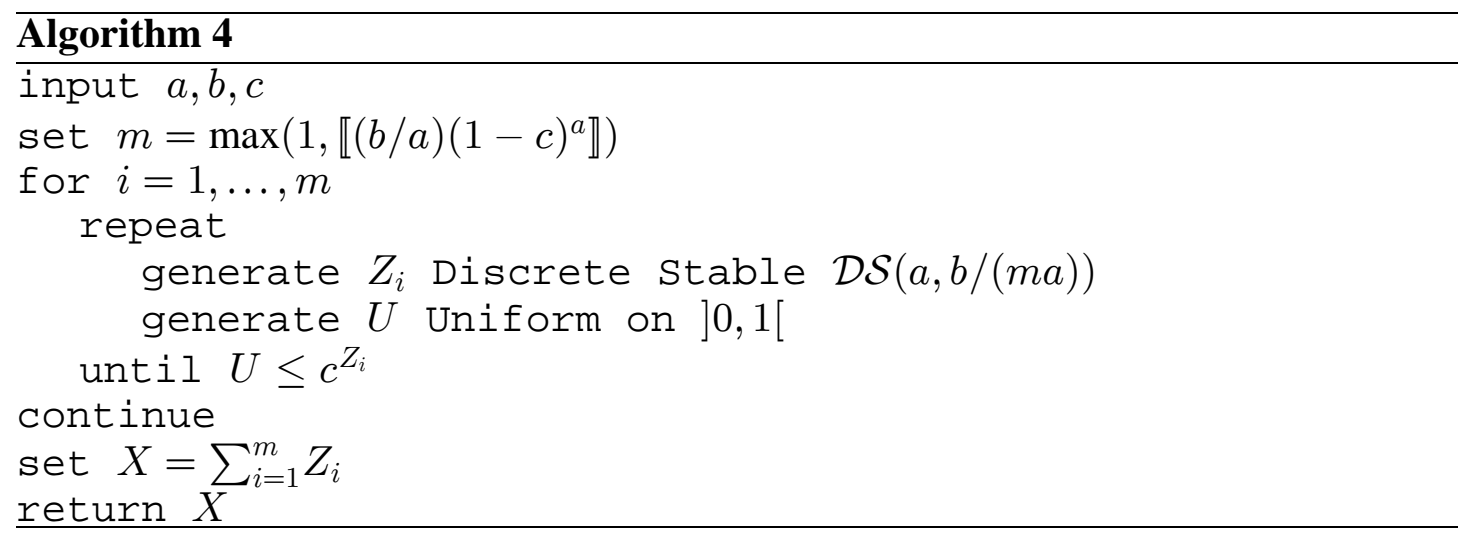

It should be remarked that $A_{\mathrm{IN}}\left(m^{*}\right)=O(b / a)$, while $A_{\mathrm{IN}}\left(m^{*}\right) \leq A_{\mathrm{IN}}(1)=A_{\mathrm{N}}$. Therefore, even if Algorithm 4 always improves over Algorithm 3, in turn its performance deteriorates when $a \downarrow 0$ or $b \rightarrow \infty$. Moreover, on the basis of the considerations carried out 
for Algorithm 3, it should be remarked that Algorithm 4 involves an average of $A_{\mathrm{IN}}\left(m^{*}\right)$ Poisson variates, $2 A_{\mathrm{IN}}\left(m^{*}\right)$ Uniform variates and $A_{\mathrm{IN}}\left(m^{*}\right)$ Exponential variates.

A further algorithm could be implemented by considering a different acceptance-rejection technique. Barabesi and Pratelli (2014b, 2015) provides a universal algorithm which is likely to conjugate efficiency and simplicity if applied to the Poisson-Tweedie distribution for $a \in] 0,1]$ and $c \neq 1$. In this case, by following Barabesi and Pratelli (2015), let us consider the function

$$
\alpha(q)=\frac{e^{\frac{b}{a}(1-c)^{a}}}{\pi} \int_{0}^{\pi}\left|\mathrm{E}\left[q^{X} e^{\mathrm{i} t X}\right]\right| \mathrm{d} t=\frac{e^{\frac{b}{a}(1-c)^{a}}}{\pi} \int_{0}^{\pi} \exp \left[-b \rho_{a, c q}(t) \cos \left(\psi_{a, c q}(t)\right)\right] \mathrm{d} t,
$$

where $\rho_{a, d}$ and $\psi_{a, d}$ are introduced in Section 3. It should be also remarked that $\alpha(q)$ is defined for $q \in] 0,1 / c]$. Moreover, by assuming that $\alpha_{1}=\alpha\left(q_{1}\right), \alpha_{2}=\alpha\left(q_{2}^{-1}\right)$ and $\nu=\alpha(1)$ for the sake of simplicity, let us denote by

$$
\beta_{1}=\min \left(\left\lfloor\log _{q_{1} q_{2}}\left(\alpha_{1} / \alpha_{2}\right)\right\rfloor,\left\lfloor\log _{q_{1}}\left(\alpha_{1} / \nu\right)\right\rfloor\right)
$$

and

$$
\beta_{2}=\max \left(\left\lfloor\log _{q_{1} q_{2}}\left(\alpha_{1} / \alpha_{2}\right)\right\rfloor+1,\left\lceil\log _{q_{2}}\left(\nu / \alpha_{2}\right)\right\rceil\right),
$$

where $\lfloor\cdot\rfloor$ and $\lceil\cdot\rceil$ represent the floor function and the ceiling function, respectively. Moreover, let us consider the quantities

$$
\omega_{1}=\frac{\alpha_{1} q_{1}^{-\beta_{1}}}{A\left(1-q_{1}\right)}, \omega_{2}=\frac{\alpha_{2} q_{2}^{\beta_{2}}}{A\left(1-q_{2}\right)}, \omega_{3}=\frac{\nu\left(\beta_{2}-\beta_{1}-1\right)}{A},
$$

and

$$
A_{\mathrm{BP}}\left(q_{1}, q_{2}\right)=\frac{\alpha_{1} q_{1}^{-\beta_{1}}}{1-q_{1}}+\frac{\alpha_{2} q_{2}^{\beta_{2}}}{1-q_{2}}+\nu\left(\beta_{2}-\beta_{1}-1\right) .
$$

Finally, let us choose $q_{1}$ and $q_{2}$ as $\left(q_{1}^{*}, q_{2}^{*}\right)=\arg \min A\left(q_{1}, q_{2}\right)$ - where minimization is carried out under the constrain on the domain of $\alpha(q)$. It is worth noting that in such a case $A_{\mathrm{BP}}\left(q_{1}^{*}, q_{2}^{*}\right)$ represents the rejection constant (see Barabesi and Pratelli, 2015). Thus, for the Poisson-Tweedie distribution the universal algorithm specializes to:

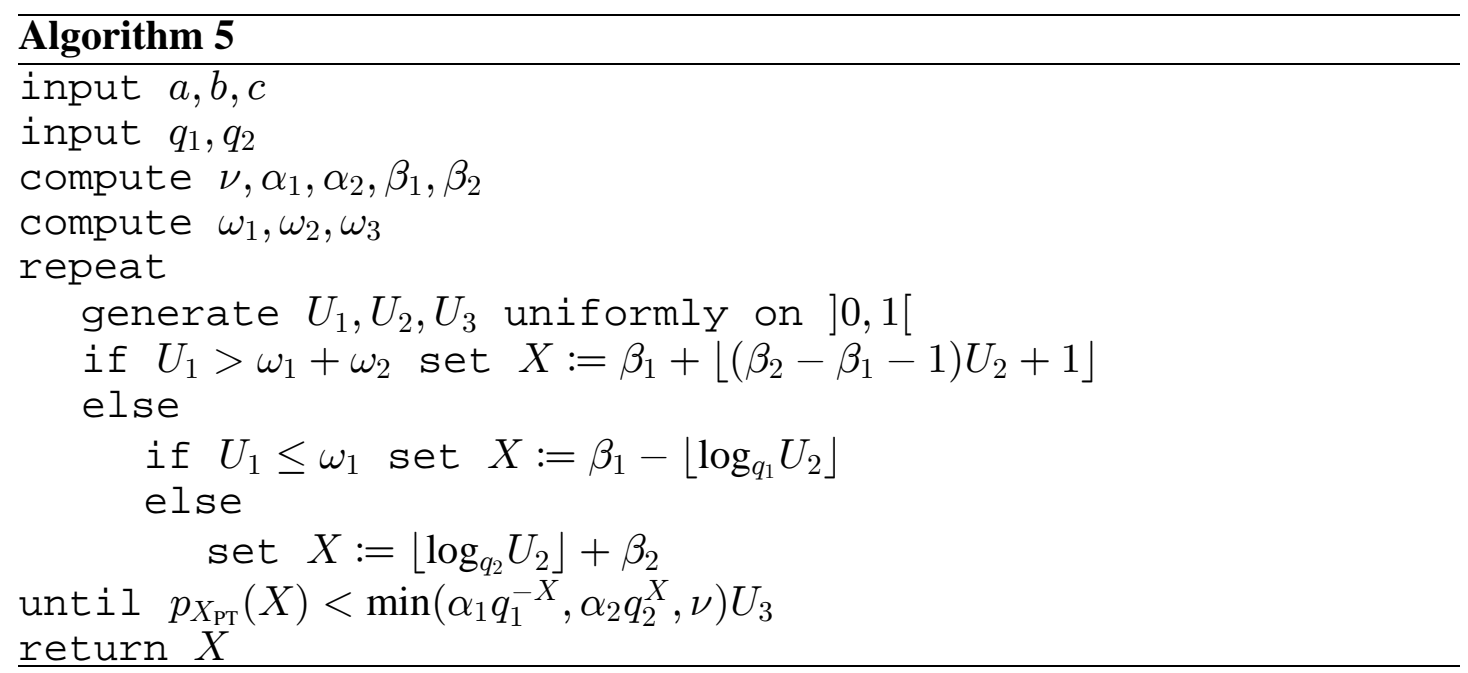


Algorithm 5 involves the computation of $p_{X_{\mathrm{PT}}}$ which may be carried out by using expression (9) or by adopting eventually expression (12). In any case, in order to avoid the complete evaluation of $p_{X_{\mathrm{PT}}}$, the approximation (10) could be considered for large arguments. Finally, it should be remarked that Algorithm 5 involves an average of $3 A_{\mathrm{BP}}\left(q_{1}^{*}, q_{2}^{*}\right)$ Uniform variates.

In order to assess the practical performance of the considered algorithms (except than Algorithm 3 which is obviously too inefficient), we considered some studies for selected values of the parameters $a, b$ and $c$. Since the four algorithms are quite rather different in their own genesis (Algorithm 1 and 2 originate from stochastic representations, while Algorithms 4 and 5 stem from the acceptance-rejection method), at first we opted to compare Algorithms 4 and 5 on the basis of the values of the rejection constant. The results of the study were reported in Table I. The analysis of Table I shows that Algorithm 5 is usually better than Algorithm 4, except few cases when $c$ is equal to 0.9. A large (not reported) study has shown that Algorithm 5 is generally more efficient than Algorithm 4 and its the performance increases as $b$ increases. Hence, Algorithm 4 could be solely suitable for small $b$ and large $a$. Incidentally, it is worth noting that the former algorithm proposed by Barabesi and Pratelli (2014b) for the generation of Poisson-Tweedie variates is slightly less efficient than Algorithm 5 in term of the rejection constant (see Table 3 in their paper) - indeed, exponential tails produces a better fit than quadratic tails in the acceptance-rejection method.

Table I. Rejection constants $A_{\mathrm{IN}}\left(m^{*}\right)$ and $A_{\mathrm{BP}}\left(q_{1}^{*}, q_{2}^{*}\right)$ for Algorithm 4 and Algorithm 5 .

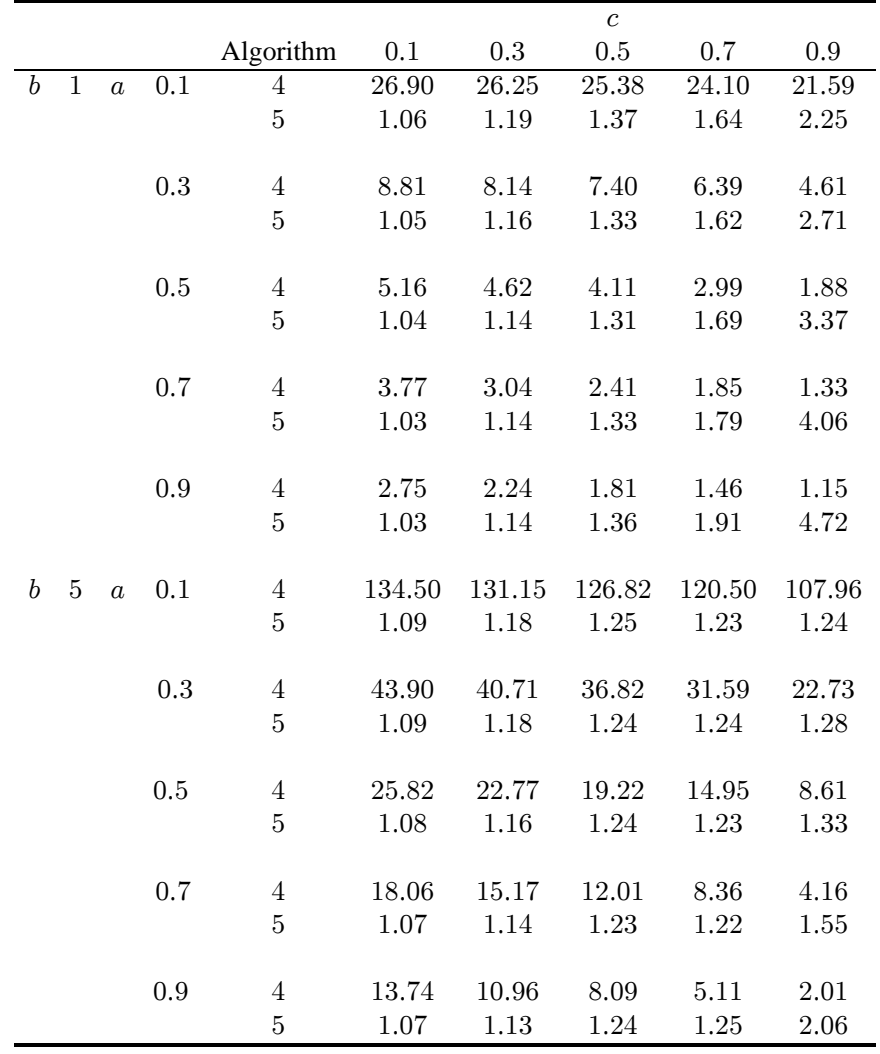


Table II. Timings (in seconds) needed for the generation of 1000 variates.

\begin{tabular}{|c|c|c|c|c|c|c|c|c|c|}
\hline & & & & \multirow[b]{2}{*}{ Algorithm } & \multicolumn{5}{|c|}{$c$} \\
\hline & & & & & 0.1 & 0.3 & 0.5 & 0.7 & 0.9 \\
\hline \multirow[t]{25}{*}{$b$} & 1 & $a$ & 0.1 & 1D & 0.66 & 0.70 & 0.73 & 0.77 & 0.89 \\
\hline & & & & $1 \mathrm{H}$ & 0.92 & 0.92 & 0.91 & 0.92 & 0.93 \\
\hline & & & & 2 & 0.70 & 0.69 & 0.67 & 0.67 & 0.67 \\
\hline & & & & 4 & 41.50 & 40.72 & 42.48 & 40.01 & 38.74 \\
\hline & & & & 5 & 0.06 & 0.06 & 0.13 & 0.28 & 2.95 \\
\hline & & & 0.3 & 1D & 0.66 & 0.66 & 0.70 & 0.72 & 0.75 \\
\hline & & & & $1 \mathrm{H}$ & 0.38 & 0.36 & 0.36 & 0.36 & 0.38 \\
\hline & & & & 2 & 0.34 & 0.34 & 0.33 & 0.34 & 0.34 \\
\hline & & & & 4 & 4.88 & 4.50 & 4.07 & 4.77 & 3.37 \\
\hline & & & & 5 & 0.06 & 0.08 & 0.11 & 0.25 & 3.38 \\
\hline & & & 0.5 & 1D & 0.64 & 0.64 & 0.66 & 0.66 & 0.63 \\
\hline & & & & $1 \mathrm{H}$ & 0.25 & 0.27 & 0.27 & 0.27 & 0.27 \\
\hline & & & & 2 & 0.23 & 0.23 & 0.23 & 0.23 & 0.24 \\
\hline & & & & 4 & 1.44 & 1.23 & 1.69 & 1.23 & 0.78 \\
\hline & & & & 5 & 0.05 & 0.07 & 0.11 & 0.25 & 3.51 \\
\hline & & & 0.7 & 1D & 0.58 & 0.58 & 0.56 & 0.55 & 0.50 \\
\hline & & & & $1 \mathrm{H}$ & 0.20 & 0.20 & 0.20 & 0.20 & 0.22 \\
\hline & & & & 2 & 0.20 & 0.20 & 0.20 & 0.20 & 0.20 \\
\hline & & & & 4 & 0.92 & 0.73 & 0.56 & 0.45 & 0.31 \\
\hline & & & & 5 & 0.05 & 0.08 & 0.11 & 0.27 & 4.66 \\
\hline & & & 0.9 & 1D & 0.48 & 0.45 & 0.45 & 0.42 & 0.36 \\
\hline & & & & $1 \mathrm{H}$ & 0.17 & 0.17 & 0.17 & 0.17 & 0.19 \\
\hline & & & & 2 & 0.17 & 0.17 & 0.17 & 0.17 & 0.17 \\
\hline & & & & 4 & 0.48 & 0.39 & 0.31 & 0.28 & 0.20 \\
\hline & & & & 5 & 0.05 & 0.06 & 0.11 & 0.27 & 5.56 \\
\hline \multirow[t]{25}{*}{$b$} & 5 & $a$ & 0.1 & 1D & 0.42 & 0.50 & 0.57 & 0.66 & 0.83 \\
\hline & & & & $1 \mathrm{H}$ & 4.08 & 4.15 & 4.04 & 4.04 & 3.79 \\
\hline & & & & 2 & 2.05 & 2.07 & 1.89 & 2.09 & 1.95 \\
\hline & & & & 4 & 217.15 & 207.62 & 203.77 & 202.08 & 191.87 \\
\hline & & & & 5 & 0.07 & 0.15 & 0.35 & 1.13 & 1.24 \\
\hline & & & 0.3 & 1D & 0.41 & 0.49 & 0.58 & 0.71 & 0.86 \\
\hline & & & & $1 \mathrm{H}$ & 1.46 & 1.40 & 1.33 & 1.31 & 1.17 \\
\hline & & & & 2 & 0.87 & 0.93 & 0.86 & 0.87 & 0.84 \\
\hline & & & & 4 & 23.87 & 23.45 & 22.29 & 21.33 & 20.25 \\
\hline & & & & 5 & 0.06 & 0.14 & 0.29 & 0.78 & 1.28 \\
\hline & & & 0.5 & 1D & 0.46 & 0.53 & 0.64 & 0.75 & 1.02 \\
\hline & & & & $1 \mathrm{H}$ & 0.89 & 0.87 & 0.83 & 0.77 & 0.69 \\
\hline & & & & 2 & 0.60 & 0.60 & 0.60 & 0.60 & 0.61 \\
\hline & & & & 4 & 7.35 & 7.00 & 6.29 & 5.98 & 4.76 \\
\hline & & & & 5 & 0.07 & 0.14 & 0.23 & 0.49 & 1.33 \\
\hline & & & 0.7 & 1D & 0.54 & 0.61 & 0.86 & 0.88 & 0.88 \\
\hline & & & & $1 \mathrm{H}$ & 0.67 & 0.64 & 0.61 & 0.53 & 0.49 \\
\hline & & & & 2 & 0.50 & 0.50 & 0.51 & 0.51 & 0.49 \\
\hline & & & & 4 & 3.43 & 3.15 & 3.07 & 2.46 & 2.06 \\
\hline & & & & 5 & 0.06 & 0.12 & 0.21 & 0.39 & 2.21 \\
\hline & & & 0.9 & 1D & 0.66 & 0.69 & 0.72 & 0.73 & 0.67 \\
\hline & & & & $1 \mathrm{H}$ & 0.55 & 0.51 & 0.45 & 0.42 & 0.37 \\
\hline & & & & 2 & 0.43 & 0.42 & 0.42 & 0.42 & 0.42 \\
\hline & & & & 4 & 2.29 & 2.04 & 1.68 & 1.33 & 0.72 \\
\hline & & & & 5 & 0.06 & 0.09 & 0.17 & 0.34 & 2.65 \\
\hline
\end{tabular}

As previously emphasized, owing to the different nature of the proposed algorithms, it is not possible to evaluate their performance on the basis of a unique benchmark. Hence, we decided to compare the algorithms on the basis of the time elapse in generating a set of 1000 
Poisson-Tweedie variates. The algorithms were implemented as routines by using the Mathematica software (Wolfram Research, 2008) and they were run on a personal computer.

As to the specific implementations of the algorithms, it should be remarked that Algorithm 1 strongly depends on the choice of the absolutely-continuous Tweedie variate generator. To this aim, two generators are mainly available, i.e. the algorithms introduced by Devroye (2009) and by Hofert (2011), respectively. Hence, we implemented two versions of Algorithm 1, i.e. Algorithm 1D (based on Devroye's method) and Algorithm 1H (based on Hofert's method). Algorithm 2 was implemented by using the usual simple Geometric variate generator (see e.g. Devroye, 1986, p. ?) and the Beta variate generator based on the Jöhnk (1964) method - which is the most efficient in this case since the parameters of the Beta r.v. involved in representation (6) are less than unity. Finally, as to Algorithms 1D, 1H, 2 and 4, Poisson variates were obtained by using the method proposed by Ahrens and Dieter (1982), which is implemented as a built-in routine in Mathematica.

The results of the study were reported in Table II. The analysis of this table shows that Algorithm 5 has generally the best performance except than for $c=0.9$, while Algorithm 2 is generally the most efficient in the remaining cases. Algorithm 5 is rather inefficient for $b=1$ and $c=0.9$, even if we have assessed in further (not reported) simulations that its performance markedly increases as $b$ increases also in this case. The drawback depends on the time-consuming evaluation of $p_{X_{\mathrm{PT}}}$, since the Poisson-Tweedie distribution is long-tailed as $c$ approaches unity. Algorithm 1D displays a quite steady performance and tends to be more efficient than Algorithm $1 \mathrm{H}$ for $b=5$, while the situation reverses for $b=1$. Further simulations (not reported) show that Algorithm $1 \mathrm{D}$ is preferable to Algorithm $1 \mathrm{H}$ as $b$ increases. Finally, Algorithm 4 seems generally rather inefficient.

5. An analysis of scientometric data. Over-dispersed and heavy-tailed distributions are of central relevance for bibliometric and scientometric scholars. First, Lotka (1926) presented a power-law distribution for modeling data in a paper usually considered the milestone in the bibliometric field. More precisely, Lotka investigated the frequency distribution of the number of manuscripts authored by a group of chemists and physicists. Since then a huge amount of papers has been dedicated to scientometrics. They can be grouped into two main sets based on different perspectives (Wilson, 1999).

In the first stream, a deterministic approach is adopted by considering bibliometric data as generated by a power law. Phenomena such as the number of papers produced by a single researcher or a group of researchers, the number of papers published in journals, the number of paper references are often modeled by considering a power law. Egghe (2005) suggests to label this approach as "Lotkaian Informetrics". Under this approach, a general process is considered by assuming sources (such as authors or journals) which produce items (e.g. papers and references). A power-law structure for frequency distribution is then assumed without providing some empirical evidence in support (for a discussion, see Burrell, 2014). This deterministic approach simply avoids the problem of zeroes owing to the underlying generating process - which is unable to produce null values. For instance, Lotka (1926) defines as authors the scholars listed in the bibliographic archive which he considered for his analysis - and, therefore, an author of the list had authored at least a paper. Similarly, the frequency distribution of papers - according to the number of times in which they appear in the reference list of a set of journals - has obviously a minimum value of one, since the considered papers are by definition included at least once in the reference list. These distributions are "truncated in zero by their very nature" (Schubert and Glänzel, 1984). When processes generating zero values are instead considered, the deterministic approach cannot 
handle the complete available information. As an example, this is the case of the distribution of the number of citations received by papers, which usually displays even an excess of zeroes (see e.g. the discussion provided by Baccini et al., 2014).

In the second perspective, bibliometric data are considered as the realization of an underlying stochastic process. To this aim, Wilson (1999) remarks that “... given that the regularities are considered to arise largely from probabilistic processes, the degree to which the purely deterministic inverse power law has dominated descriptions may seem surprising ...”. Accordingly, compound and mixture models have been often considered in scientometrics. As an example, Rao (1980) proposed the Negative Binomial distribution, while Sichel (1985) introduced the Generalized Inverse Gaussian Poisson distribution (see also Burrell and Fenton, 1993). However, when the major source of over-dispersion is related to an excess of zero counts, further flexible models could be helpful. Owing to the genesis described in Section 2, the Poisson-Tweedie distribution may adequately fit zero-inflated data, as well as heavy-tailed data.

In order to illustrate a scientometric application of the Poisson-Tweedie distribution, a total of $n=371$ articles published by Metron and $n=395$ articles published by Statistical Methods and Applications (whose usually-adopted acronym is SMAP). As is known, Metron and SMAP are two Italian statistical journals surveyed by the Scopus database. Data were retrieved in September 2014 from the Scopus repository and refer to the articles published in the period 1999-2014 for Metron and in the period 2001-2014 for SMAP. The observed frequencies $n_{k}$ of the so-called "citation profile" were considered for both the journals and the results were reported in Table II. For the sake of clarity, $n_{k}$ actually represents the number of published papers with $k$ citations. On the basis of the observed frequencies, the maximum-likelihood estimates of the Poisson-Tweedie parameters were obtained for each journal. More precisely, these estimates were $\widehat{a}=0.304(0.037), \widehat{b}=0.463(0.031)$ and $\widehat{c}=0.902(0.012) \quad$ for Metron and $\widehat{a}=0.263(0.034), \quad \widehat{b}=0.513(0.032) \quad$ and $\widehat{c}=0.909(0.009)$ for SMAP (standard deviations in parenthesis). The corresponding estimated frequencies were reported in Table II. These values emphasize the excellent agreement of the estimated frequencies to the observed frequencies. Indeed, the $\chi^{2}$ statistic turns out to be 6.07 for Metron data and 17.03 for SMAP data. In contrast, even if the results of this further study are not reported, we have assessed that the Generalized Inverse Gaussian Poisson distribution does not adequately fit the same data. Incidentally, it is worth remarking that estimates for the two journals are quite similar, showing that the two distributions are quite heavy-tailed (the estimates of $c$ are close to one - the value for which the PoissonTweedie reduces to a Discrete Stable model). On the basis of this specific analysis, we argue that the Poisson-Tweedie distribution could find an interesting use also in the scientometric framework.

Table III. Observed and fitted citation distribution for Metron and SMAP. 


\begin{tabular}{cccccc}
\hline \multicolumn{3}{c}{ Metron } & \multicolumn{3}{c}{ SMAP } \\
$k$ & $n_{k}$ & $n p_{X}(k)$ & $k$ & $n_{k}$ & $n p_{X}(k)$ \\
\hline 0 & 172 & 171.28 & 0 & 160 & 158.87 \\
1 & 69 & 71.59 & 1 & 68 & 74.08 \\
2 & 38 & 37.46 & 2 & 48 & 42.06 \\
3 & 23 & 22.97 & 3 & 28 & 27.28 \\
4 & 17 & 15.44 & 4 & 18 & 19.12 \\
5 & 12 & 11.00 & 5 & 17 & 14.08 \\
6 & 8 & 8.14 & 6 & 8 & 10.72 \\
7 & 5 & 6.20 & 7 & 13 & 8.37 \\
8 & 6 & 4.82 & 8 & 5 & 6.65 \\
9 & 4 & 3.81 & 9 & 3 & 5.36 \\
10 & 5 & 3.05 & 10 & 3 & 4.37 \\
11 & 2 & 2.47 & 11 & 1 & 3.60 \\
12 & 3 & 2.02 & 12 & 6 & 2.99 \\
13 & 1 & 1.66 & 13 & 2 & 2.50 \\
14 & 2 & 1.38 & 14 & 1 & 2.10 \\
15 & 0 & 1.15 & 15 & 2 & 1.77 \\
16 & 0 & 0.96 & 16 & 1 & 1.50 \\
17 & 1 & 0.81 & 17 & 0 & 1.28 \\
$>17$ & 3 & 4.80 & 18 & 1 & 1.09 \\
& & & 19 & 1 & 0.94 \\
& & & 20 & 0 & 0.70 \\
& & & 21 & 1 & 0.60 \\
& & & $>21$ & 8 & 4.98 \\
\hline
\end{tabular}

As to the computational issues involved in the evaluation of the maximum-likelihood estimates of the Poisson-Tweedie parameters, it should be remarked that the likelihood function was computed by means of both expression (9) and expression (12). The use of the two expressions led to nearly identical values and did not produce numerical drawbacks. In order to obtain a further validation of the results, the maximum-likelihood estimates were also computed by means of the software package tweeDEseq for R proposed by Esnaola et al. (2013). The package adopts the recursive expression provided by El-Shaarawi et al. (2011) in order to compute $p_{X_{\mathrm{PT}}}$. In turn, the results were in agreement with those computed on the basis of expressions (9) and (12).

Finally, a further experiment was implemented in order to assess the quality of the variates obtained by using the considered algorithms. Indeed, we generated the Poisson-Tweedie variates by using the maximum-likelihood estimates of the Metron and SMAP data as the values for the parameters $a, b$ and $c$-i.e. values which are likely to occur in a real scenario. The quality of the variates generated according to the algorithms considered in Table II was verified on the basis of some empirical indexes which are compared with the corresponding true model indexes - i.e. the mean, the standard deviation and the skewness and kurtosis coefficients, which are denoted by means of the symbols $\mu, \sigma, \alpha_{3}$ and $\alpha_{4}$, respectively. The closed expressions for $\mu, \sigma, \alpha_{3}$ and $\alpha_{4}$ in terms of $a, b$ and $c$ may be easily found by differentiating $G_{X_{\mathrm{PT}}}$ (see e.g. El-Shaarawi et al., 2011). The empirical indexes were computed on the basis of $1,000,10,000$ and 100,000 variates generated by means of each algorithm and the output was reported in Table IV. In addition, the $\chi^{2}$ statistic was also computed for the same sets of variates (by grouping into 20 cells, similarly to Table III) and it was reported in Table IV. By analyzing this table, it can be concluded that the quality of the generated variates is satisfactory for the considered algorithms. 
Table IV. Quality assessment of the generated variates for the considered algorithms.

Empirical indexes are computed on the basis of 1,000(10,000)100, 000 replicates.

\begin{tabular}{ccccccccc}
\hline$a$ & $b$ & $c$ & Algorithm & \multicolumn{5}{c}{} \\
\hline 0.304 & 0.463 & 0.902 & & $\mu=2.10$ & $\sigma=3.95$ & $\alpha_{3}=4.11$ & $\alpha_{4}=30.11$ & $\chi^{2}$ \\
& & & $1 \mathrm{D}$ & $2.22(2.18) 2.12$ & $3.95(4.08) 3.94$ & $3.49(4.07) 4.01$ & $19.74(28.51) 28.18$ & $12.65(18.18) 23.44$ \\
& & & $1 \mathrm{H}$ & $2.15(2.09) 2.10$ & $4.17(3.93) 3.96$ & $3.98(4.24) 4.17$ & $24.92(31.57) 30.74$ & $16.48(18.15) 12.41$ \\
& & & 2 & $2.14(2.11) 2.10$ & $3.87(3.93) 3.95$ & $3.74(3.70) 4.09$ & $25.11(21.74) 29.20$ & $23.38(18.37) 16.98$ \\
& & & 4 & $2.26(2.12) 2.10$ & $4.02(3.89) 3.93$ & $3.78(4.02) 4.09$ & $24.35(31.15) 30.03$ & $22.13(23.94) 18.66$ \\
& & & 5 & $2.02(2.10) 2.10$ & $3.82(4.00) 3.95$ & $4.38(4.13) 4.11$ & $32.50(28.20) 30.22$ & $22.66(18.31) 23.07$ \\
0.263 & 0.513 & 0.909 & & & & & & \\
& & & $1 \mathrm{D}$ & $2.68(2.73) 2.73$ & $4.27(4.67) 4.76$ & $2.78(3.43) 3.70$ & $12.84(19.81) 23.94$ & $16.36(20.91) 21.56$ \\
& & & $1 \mathrm{H}$ & $2.55(2.75) 2.74$ & $4.45(4.90) 4.82$ & $3.89(3.81) 3.73$ & $26.17(25.27) 24.70$ & $20.56(27.20) 18.65$ \\
& & & 2 & $2.69(2.73) 2.72$ & $4.60(4.81) 4.77$ & $3.26(3.73) 3.80$ & $16.88(24.32) 25.13$ & $10.98(16.95) 23.67$ \\
& & & 4 & $2.78(2.84) 2.73$ & $4.97(5.01) 4.78$ & $4.87(3.93) 3.65$ & $42.48(27.04) 23.30$ & $22.30(18.99) 22.46$ \\
& & & & $2.56(2.73) 2.73$ & $4.36(4.86) 4.81$ & $3.77(3.92) 3.79$ & $25.33(27.15) 25.27$ & $15.34(23.81) 21.30$ \\
\hline
\end{tabular}

\section{Acknowledgements}

The authors would like to thank the two anonymous reviewers for their valuable comments and suggestions which have improved the early version of the paper.

\section{References}

Aalen, O.O. (1992) Modelling heterogeneity in survival analysis by the compound Poisson distribution, Annals of Applied Probability 2, 951-972.

Ahrens, J. H. and Dieter, U. (1982) Computer generation of Poisson deviates from modified Normal distributions, ACM Transactions on Mathematical Software 8, 163-179.

Baccini, A., Barabesi, L., Cioni, M. and Pisani, C. (2014) Crossing the hurdle: the determinants of individual scientific performance, Scientometrics 101, 2035-2062.

Barabesi, L. and Pratelli, L. (2014a) Discussion of "On simulation and properties of the Stable law” by L. Devroye and L. James, Statistical Methods and Applications 23, 345351.

Barabesi, L. and Pratelli, L. (2014b) A note on a universal random variate generator for integer-valued random variables, Statistics and Computing 24, 589-596.

Barabesi, L. and Pratelli, L. (2015) Universal methods for generating random variables with a given characteristic function, Journal of Statistical Computation and Simulation 85, 16791691.

Burrell, Q.L. (2014) The individual author's publication-citation process: theory and practice, Scientometrics 98, 725-742.

Burrell, Q.L. and Fenton, M.R. (1993) Yes, the GIGP really does work - and is workable!, Journal of the American Society for Information Science 44, 61-69.

Devroye, L. (1986) Non-uniform random variate generation, Springer, New York.

Devroye, L. (1993) A triptych of discrete distribution related to the stable law, Statistics and Probability Letters 18, 349-351.

Devroye, L. (2009) Random variate generation for exponentially and polynomially tilted stable distributions, ACM Transactions on Modeling and Computer Simulation 19, Article 18.

Dunn, P.K. and Smyth, G.K. (2008) Evaluation of Tweedie exponential dispersion model densities by Fourier inversion, Statistics and Computing 18, 73-86.

Egghe, L. (2005) Power laws in the information production process: Lotkaian informetrics, Elsevier, Oxford. 
El-Shaarawi, A.H., Zhu, R. and Joe, H. (2011) Modelling species abundance using the Poisson-Tweedie family, Environmetrics 22, 152-164.

Esnaola, M., Puig, P., Gonzalez, D., Castelo, R. and Gonzalez, J.R. (2013) A flexible count data model to fit the wide diversity of expression profiles arising from extensively replicated RNA-seq experiments, BMC Bioinformatics 14, 254.

Gerber, H.U. (1991) From the generalized gamma to the generalized negative binomial distribution, Insurance: Mathematics and Economics 10, 303-309.

Hao-Chun Chuang, H. and Oliva, R. (2014) Estimating retail demand with Poisson mixtures and out-of-sample likelihood, Applied Stochastic Models in Business and Industry 30, 455-463.

Hofert, M. (2011) Sampling exponentially tilted stable distributions, ACM Transactions on Modeling and Computer Simulation 22, Article 3.

Hougaard, P. (1986) Survival models for heterogeneous populations derived from stable distributions, Biometrika 73, 387-396.

Hougaard, P., Lee M.T. and Whitmore, G.A. (1997) Analysis of overdispersed count data by mixtures of Poisson variables and Poisson processes, Biometrics 53, 1225-1238.

Johnson, N.L., Kemp, A.W. and Kotz, S. (2005) Univariate discrete distributions, 3rd edn., Wiley, New York.

Jöhnk, M.D. (1975) Erzeugung von betaverteilten und gammaverteilten Zufallszahlen, Metrika 8, 5-15.

Kanter, M. (1975) Stable densities under change of scale and total variation inequalities, Annals of Probability 3, 697-707.

Kokonendji, C.C., Dossou-Gbété S. and Demétrio, C.G.B. (2004) Some discrete exponential dispersion models: Poisson-Tweedie and Hinde-Demétrio classes, SORT 28, 201-214.

Lotka, A.J. (1926) The frequency distribution of scientific productivity, Journal of the Washington Academy of Sciences 16, 317-323.

Marcheselli, M., Baccini, A. and Barabesi, L. (2008) Parameter estimation for the discrete stable family, Communications in Statistics - Theory and Methods 37, 815-830.

Rao, I.K.R. (1980) The distribution of scientific productivity and social change, Journal of the American Society for Information Science 31, 111-122.

Schubert, A. and Glänzel, W. (1984) A dynamic look at a class of skew distributions. A model with scientometric applications, Scientometrics 6, 149-167.

Sibuya, M. (1979) Generalized hypergeometric, digamma and trigamma distributions, Annals of the Institute of Statistical Mathematics 31, 373-390.

Sichel, H.S. (1985) A bibliometric distribution which really works, Journal of the American Society for Information Science 36, 314-321.

Tweedie, M.C.K. (1984) An index which distinguishes between some important exponential families, in Statistics: Applications and New Directions, Proceedings of the Indian Statistical Institute Golden Jubilee International Conference (J.K. Ghosh and J. Roy, eds.), Indian Statistical Institute, Calcutta, pp. 579-604.

Wilson, C.S. (1999) Informetrics, Annual Review of Information Science and Technology 34, 107-247.

Wolfram Research, Inc. (2008) Mathematica, Version 7.0, Champaign, Illinois.

Zhu, R. and Joe, H. (2009) Modelling heavy-tailed count data using a generalized Poissoninverse Gaussian family, Statistics \& Probability Letters 79, 1695-1703.

\section{Appendix}


Result 1. By expanding (1) in exponential and binomial series, it follows that

$$
\begin{aligned}
G_{X_{\mathrm{PT}}}(s) & =e^{\frac{b}{a}(1-c)^{a}} \sum_{m=0}^{\infty}(-1)^{m} \frac{(b / a)^{m}}{m !}(1-c s)^{a m} \\
& =e^{\frac{b}{a}(1-c)^{a}} \sum_{m=0}^{\infty}(-1)^{m} \frac{(b / a)^{m}}{m !} \sum_{k=0}^{\infty}\left(\begin{array}{c}
a m \\
k
\end{array}\right)(-c s)^{k} \\
& =e^{\frac{b}{a}(1-c)^{a}} \sum_{k=0}^{\infty}(-c s)^{k} \sum_{m=0}^{\infty}(-1)^{m}\left(\begin{array}{c}
a m \\
k
\end{array}\right) \frac{(b / a)^{m}}{m !}
\end{aligned}
$$

and hence

$$
p_{X_{\mathrm{PT}}}(k)=e^{\frac{b}{a}(1-c)^{a}}(-c)^{k} \sum_{m=0}^{\infty}(-1)^{m}\left(\begin{array}{c}
a m \\
k
\end{array}\right) \frac{(b / a)^{m}}{m !} I_{\mathbb{N}}(k) .
$$

Moreover, by using the straightforward identity $\left(\begin{array}{c}a m \\ k\end{array}\right)=\left.\frac{1}{k !} \frac{d^{k} x^{a m}}{d x^{k}}\right|_{x=1}$, it also holds that

$$
\begin{aligned}
\sum_{m=0}^{\infty}(-1)^{m}\left(\begin{array}{c}
a m \\
k
\end{array}\right) \frac{(b / a)^{m}}{m !} & =\left.\frac{1}{k !} \frac{d^{k} e^{-\frac{b}{a} x^{a}}}{d x^{k}}\right|_{x=1}=\left.\frac{e^{-\frac{b}{a}}}{k !} \frac{d^{k} e^{\frac{b}{a}\left(1-x^{a}\right)}}{d x^{k}}\right|_{x=1} \\
& =\left.\frac{e^{-\frac{b}{a}}}{k !} \sum_{m=0}^{k} \frac{(b / a)^{m}}{m !} \frac{d^{k}\left(1-x^{a}\right)^{m}}{d x^{k}}\right|_{x=1} \\
& =e^{-\frac{b}{a}} \sum_{m=0}^{k} \frac{(b / a)^{m}}{m !} \sum_{j=0}^{m}(-1)^{j}\left(\begin{array}{c}
m \\
j
\end{array}\right)\left(\begin{array}{c}
a j \\
k
\end{array}\right),
\end{aligned}
$$

since $\left.\frac{d^{k}\left(1-x^{a}\right)^{m}}{d x^{k}}\right|_{x=1}=0$ when $k>m$. Thus, expression (9) promptly follows.

Result 2. On the basis of the expression of $p_{X_{\mathrm{PT}}}$ given in Result 1, it follows

$$
G_{X_{\mathrm{PT}}}(s)=e^{\frac{b}{a}\left[(1-c)^{a}+1\right]} \sum_{k=0}^{\infty}(-c s)^{k} \mathrm{E}\left[(-1)^{M}\left(\begin{array}{c}
a M \\
k
\end{array}\right)\right],
$$

where $M$ represents a $\mathcal{P}(b / a)$ r.v. Hence, it also holds that

$$
p_{X_{\mathrm{PT}}}(k)=e^{\frac{b}{a}\left[(1-c)^{a}+1\right]}(-c)^{k} \mathrm{E}\left[(-1)^{M}\left(\begin{array}{c}
a M \\
k
\end{array}\right)\right] .
$$

Therefore, for $a \in] 0,1]$, from the previous expression we obtain

$$
\begin{aligned}
p_{X_{\mathrm{PT}}}(k) & \leq e^{\frac{b}{a}\left[(1-c)^{a}+1\right]} c^{k} \mathrm{E}\left[\left|\left(\begin{array}{c}
a M \\
k
\end{array}\right)\right|\right] \\
& \leq \frac{b}{a}\left(1+\frac{b}{a}\right) e^{\frac{b}{a}\left[(1-c)^{a}+1\right]} c^{k} k^{-a-1} .
\end{aligned}
$$




\section{Reply to Associate Editor}

Dear Associate Editor,

we have modified the first draft of the manuscript according to the comments of the two referees. As you have suggested, in addition to the minor changes proposed by the first referee, we have especially considered the major requirements of the second referee. We hope we have addressed the referees' suggestions satisfactorily.

Best regards

Alberto Baccini, Lucio Barabesi e Luisa Stracqualursi 


\section{Reply to Referee 1}

Comment 1. In the derivation of equation 9, the authors subtracted 1 and added 1 then expanded their generating function. This is really not needed at all if binomial expansion is done directly and then changing the order of summation so the results can be directly obtained and there are no needs for Results I in the appendix. This way no needs for the many

lines of equations given with the addition of better clarification of the results.

Reply. Thank you for your suggestion on this subtle issue. According to your comment, we have modified the initial part of Section 3 by also avoiding the use of the quantities $w_{a, m}(k)$. In order to achieve a clearer (and shorter) exposition in the main text of Section 3, we have decided to postpone the expansion in exponential and binomial series in Result 1 of the Appendix, which - even if is kept - turns out to be much shorter in the new version. Obviously, in Result 1, we also needed a passage in order to show that the summations in the expression of the p.f. $p_{X_{\mathrm{PT}}}(k)$ are finite. Incidentally, on the basis of the expansion you suggested, we have also realized that the inequality in Result 2 could be improved and a simple and accurate approximation of $p_{X_{\mathrm{PT}}}(k)$ for large $k$ could be obtained. Thus, Result 2 is in turn modified and shortened.

Comment 2. The second is with applications one does not just go ahead to apply such a model without considering the factors that led to the observed frequency. It seems to me that the large number of zeros is related to the number of years since the publication appeared. One can infer that a recently published paper will belong to zero class. I suggest adding another column with average number of years since publication as a covariate would likely improve the fit of the model and even may not need this heavy computation.

Reply.

We hope we have addressed your suggestions satisfactorily.

Thank you for your revision.

Alberto Baccini, Lucio Barabesi e Luisa Stracqualursi 


\section{Reply to Referee 2}

General Comment. The authors introduce the stochastic genesis of Poisson-Tweedie distribution and suggest several algorithms for generating the Poisson-Tweedie variates. The authors also give two scientometric data, Metron and SMAP, and state, by the excellent agreement of the estimated frequencies and observed frequencies, that that the two data do follow the Poisson-Tweedie distributions. However, the materials present in the manuscript looks not complete, since the last section (5. An analysis of scientometric data) of the manuscript seems not related to the main topic of the manuscript - the five algorithms that generate the Poisson-Tweedie variates. The readers who read the paper would expect to see stuff like, based on the collected data, quality of variates generated by the five algorithms so that the performance of the algorithms can be further compared. One way to achieve this goal is to assume the scientometric data do follow the Poisson-Tweedie distributions, and generates the Poisson-Tweedie variates using the MLEs of $a, b$, and $c$.

Reply. On the basis of your comments, we have realized that Section 5 is not homogeneous with the other parts of the manuscript. Obviously, as you surely grasp, this Section was introduced in the manuscript since our interest in the Poisson-Tweedie law originated from the need of a flexible model able to fit data which may be eventually zero-inflated or heavytailed - indeed, one of our aim consist in persuading practitioners to adopt this model which may be very suitable in scientometrics for this reasons. Thus, on the basis of your suggestion, we have largely modified Section 5 (see the final part of this section). In primis, we have emphasized that the results given in Section 3 - dealing with the expressions of the p.f. $p_{X_{\mathrm{PT}}}(k)$ - may provide a suitable computation of the maximum likelihood estimates of the parameters (in this way, the link between Section 3 and Section 5 is more apparent). Subsequently, as you recommend, we have generated the Poisson-Tweedie variates by using the maximum likelihood estimates as the values for the parameters $a, b$ and $c$. The quality of the variates (generated according the considered algorithms) has been assessed on the basis of some empirical indexes which are compared with the corresponding true model indexes (i.e. the mean, the variance and the skewness and kurtosis coefficients). In addition, the $\chi^{2}$ statistic was also computed for the same sets of test variates. Hence, the connection between Section 4 and Section 5 should be clearer.

Specific comment 1. The authors apply rejection constants as the criterion in evaluating the performance of the Algorithms 1, 4, and 5, but it is not so obvious what the rejection constants are. The authors need to have a clearer definition. Also, for Algorithm 1, we have hard time in figuring out how rejection constant plays a role in the performance evaluation. A little bit detail seems necessary. Besides rejection constants, the time elapse in generating the Poisson-Tweedie variates and the quality of the generated data should be evaluated, too.

Reply. We agree with the referee. Indeed, we attempted to present in a unique table some performance benchmarks for algorithms which are too different in their own genesis. As a matter of fact, Algorithm 1 is actually based on a stochastic representation - i.e. expression (3) of our paper - involving the generation of a Poisson variate and a Tweedie variate. Regrettably, the rejection constant reported in Table I of the previous version of the paper is solely referred to the complex algorithm proposed by Devroye (2009) adopted for the generation of the Tweedie variate - in addition, this algorithm stems from the double rejection method for which is even difficult to define the rejection constant in comparison with the usual acceptance-rejection method (indeed, we computed this constant by simulation). Furthermore, Algorithm 2 is in turn based on a rather complex stochastic representation - i.e. expression (8) of our paper - involving a (Poisson) stochastic sum of 
functions of Geometric and Beta random variables. Hence, the adopted performance benchmark - i.e. the expected number of cycles in the stochastic sum - does not adequately inform on the complexity of the algorithm. In contrast, Algorithm 4 and Algorithm 5 are actually based on the acceptance-rejection method and - more correctly - they may be judged on the basis of the rejection constants. In such a case, we opted to compare solely Algorithm 4 and Algorithm 5 on the basis of such constants and accordingly we modified Table I. By following your suggestion, we decided to compare the algorithms on the basis of the time elapse in generating the Poisson-Tweedie variates. With this aim, we tried to implement the algorithms as more efficiently as possible (we adopted the Mathematica software in so doing) and we reported the results in Table II of the new version of the manuscript. Hence, as you can see, also Section 4 was quite radically modified. Finally, by following your comment, we also evaluated the quality of the generated variates in Section 5 of the new version of the manuscript (see our reply to the general comment).

Specific comment 2. The expected number of cycles are calculated for Algorithm 2. Why the expected number, as compared to rejection constants, is a reasonable choice?

Reply. As remarked in our reply to the specific comment 1, we decided to avoid the comparison on the basis of this benchmark since it was not suitable.

Specific comment 3. For performance comparison in Table 1, people might expect to see the value of $N$, and the number of Poisson-Tweedie variates generated.

Reply. As remarked in our reply to the specific comment 1, in the new version of the manuscript, Table I solely contains the values of the rejection constant of Algorithm 4 and Algorithm 5 - which are computed on the basis of their closed expressions, i.e. by means of the values of $A_{\mathrm{IN}}\left(m^{*}\right)$ and $A_{\mathrm{BP}}\left(q_{1}^{*}, q_{2}^{*}\right)$.

Specific comment 4. From page 11, the authors state that Algorithm 5 is usually the best, except few cases for $b=1$ and $a$ or $c$ are equal to 0.9. With $c$ values for Metron and SMAP being 0.902 and 0.909 , respectively, do the authors have any comments on these cases?

Reply. Actually, we put too emphasis on the performance of Algorithm 5. In the new version of the manuscript we have modified our comments in Section 4.

We hope we have addressed your suggestions satisfactorily.

Thank you for your revision.

Alberto Baccini, Lucio Barabesi e Luisa Stracqualursi 